\title{
Power functional theory for the dynamic test particle limit
}

\author{
Joseph M Brader ${ }^{1}$ and Matthias Schmidt ${ }^{2}$ \\ ${ }^{1}$ Soft Matter Theory, University of Fribourg, Avenue de l'Europe 20, CH-1700 Fribourg, Switzerland \\ 2 Theoretische Physik II, Physikalisches Institut, Universität Bayreuth, D-95440 Bayreuth, Germany \\ E-mail: Joseph.Brader@unifr.ch and Matthias.Schmidt@uni-bayreuth.de
}

\begin{abstract}
For classical Brownian systems both in and out of equilibrium we extend the power functional formalism of Schmidt and Brader (2013 J. Chem. Phys. 138 214101) to mixtures of different types of particles. We apply the framework to develop an exact dynamical test particle theory for the self and distinct parts of the van Hove function, which characterize tagged and collective particle motion. The memory functions that induce non-Markovian dynamics are related to functional derivatives of the excess (over ideal) free power dissipation functional. The method offers an alternative to the recently found nonequilibrium Ornstein-Zernike relation for dynamic pair correlation functions.
\end{abstract}

Keywords: colloids, density functional theory, dynamics

\section{Introduction}

Most of the fundamental aspects of the dynamics of simple as well as of complex fluids can be described on the basis of microscopic two-point correlations in space and time. The collective two-point functions, such as the (total) van Hove function, the intermediate scattering function or the shear modulus [1], provide information about the relevant relaxation times at various lengthscales and enable one, via Green-Kubo relations [2,3], to calculate macroscopic transport coefficients, such as e.g. the shear viscosity. Collective correlation functions relate microstates of the system at different times with each other, treating all occuring combinations of particle pairs on the same footing. As a result, information about the (average) motion of individual particles is not resolved.

A higher level of resolution can be obtained by splitting a given collective correlation function into a sum of 'self' and 'distinct' contributions, via appropriate restriction of the occurring sum(s) over particles. The behaviour of each of the split contributions can then be studied and analyzed separately. The self contribution describes the dynamics of an arbitrarily selected (tagged) particle and yields information about e.g. the mean-squared displacement in the case of the self van Hove function [1]. Transport properties of a tagged particle, such as the self diffusion coefficient, can be obtained from integrating in time over the tagged particle velocity autocorrelation function, established via a well-known GreenKubo relation [5].
Theoretical schemes for the practical calculation of dynamic pair correlations are commonly formulated in terms of memory functions. These represent the residual influence of the unresolved microscopic degrees of freedom, which have been integrated out when formulating a closed equation of motion on the two-body level. The required coarse-graining may formally be implemented through use of the ZwanzigMori projection operator formalism [4-6]. The exact memory functions thus obtained are usually approximated using e.g. generalized hydrodynamics [7] or mode-coupling projections onto slow variables [8], in order to arrive at a closed theory. In the spirit of density functional theory a time-dependent generalization of the pair direct correlation function was also proposed some time ago [9], but remains to be tested.

Very recently, an alternative theoretical method for the determination of collective pair correlation functions in Brownian systems has been proposed by the present authors $[10,11]$. This new method is based on a nonequilibrium Ornstein-Zernike (NOZ) equation, which is a formally exact, non-Markovian equation of motion for the tensorial twobody current, $\mathrm{J}_{2}\left(\mathbf{r}_{1}, t_{1} ; \mathbf{r}_{2}, t_{2}\right)$, correlating particle current fluctuations at separate spacetime points $\mathbf{r}_{1}, t_{1}$ and $\mathbf{r}_{2}, t_{2}$. In contrast to mode-coupling-type approaches employing explicit projection operators, the statistical averaging in the NOZ theory is achieved using dynamical functional calculus [11]. From knowledge of $J_{2}$ it is straighforward to obtain the more familiar van Hove function [12] by employing the two-point 
continuity equation, and hence differentiating in space and integrating in time.

The NOZ equation can be elegantly derived within the variational framework of power functional theory (PFT) [14]. The PFT is an exact generalization of equilibrium classical density functional theory (DFT) [18] for many-body Brownian systems out-of-equilibrium. An equation of motion for the one-body current, $\mathbf{J}(\mathbf{r}, t)$, follows from minimization of a dynamic generating functional, $\mathcal{R}_{t}$, representing the 'free power' available in the system [14], and hence

$$
\frac{\delta \mathcal{R}_{t}[\rho, \mathbf{J}]}{\delta \mathbf{J}(\mathbf{r}, t)}=0
$$

where the square brackets indicate a functional dependence on the density and current fields (which both depend on space and time). It was shown that functional differentiation of the Euler-Lagrange equation (1) with respect to the external forces acting on the system yields an exact NOZ equation for the two-body current [11]. The NOZ route to the twobody correlations has the advantage over other theoretical approaches that the memory functions can be identified as functional derivatives of an excess (over ideal gas) power dissipation functional $[10,11]$, thus aiding their physical interpretation. Moreover, generating all one-body, two-body and, in principle, higher-body dynamic correlation functions from the single variational principle (1) provides a unified and internally consistent description of both the static and dynamic properties of the system.

The NOZ approach forms an analogue of the well-known static Ornstein-Zernike (OZ) relation, which is a cornerstone of the equilibrium theory of liquid structure. Within DFT the pair-direct correlation function can be generated from a second functional derivative of the excess Helmholtz free energy with respect to the one-body density distribution. The OZ relation then yields the two-body density correlator and hence the static (i.e. equal-time) pair distribution function and, via Fourier transform, the static structure factor.

An alternative, possibly less common but equally powerful route to the static structure is to minimize the grand potential functional in the presence of an external field that corresponds to a test-particle fixed at the origin. Percus' test particle idea [15] can then be used to identify the resulting one-body density profile with the two-body density correlator. Given an exact free energy functional, the $\mathrm{OZ}$ and test-particle routes to the pair correlations are formally equivalent and will thus yield identical results. In practice, if a reliable approximative free energy functional is available, then the test-particle results are often superior in quality than those from the $\mathrm{OZ}$ route, when using simulation results as benchmark data.

In this paper we develop a dynamic test particle method for obtaining dynamic pair correlations from power functional theory. The dynamical test particle method, first suggested by Archer et al [16], is straightforward to implement and provides an intuitive separation of the van Hove function into self and distinct contributions. The development of a dynamical test particle approach within power functional theory requires a multi-component formulation of the latter, which we develop in the present work. This enables us to distinguish the test particle from all other particles according to the species label. While Archer et al's implementation of the dynamic test particle concept via DDFT is approximate in nature, the use of the PFT permits us to formulate a formally exact equation of motion for the self and distinct van Hove function.

The paper is structured as follows: In section 2 we specify the microscopic dynamics and define the one-body correlations of interest. In section 3 we describe the extension of power functional theory to multicomponent systems. The self and distinct van Hove functions are introduced in section 4, and are then calculated in section 5 by applying the mixture power functional in a dynamic test particle calculation. Finally, in section 6 , we give concluding remarks and an outlook on future work.

\section{Many-body Brownian dynamics}

\subsection{Smoluchowski equation}

The system is described by a time-dependent distribution function, $\Psi\left(\mathbf{r}^{N}, t\right)$, which gives the probability density to find the $N$ particles in the system at positions $\mathbf{r}^{N} \equiv\left\{\mathbf{r}_{1}, \ldots, \mathbf{r}_{N}\right\}$ at time $t$. The total inter-particle interaction potential is $U\left(\mathbf{r}^{N}\right)$. The external force acting on a given particle $i$ consists of a conservative contribution, $-\nabla_{i} V_{i}^{\text {ext }}\left(\mathbf{r}_{i}, t\right)$, arising from an external potential (which, in principle, can be different for each particle), and a non-conservative force, $\mathbf{X}_{i}\left(\mathbf{r}_{i}, t\right)$, which could e.g. represent shear. The many-body distribution function evolves according to the Smoluchowski equation [17]

$$
\frac{\partial}{\partial t} \Psi\left(\mathbf{r}^{N}, t\right)=-\sum_{i} \nabla_{i} \cdot \hat{\mathbf{v}}_{i}(t) \Psi\left(\mathbf{r}^{N}, t\right),
$$

where the velocity operator of particle $i$ is defined as

$$
\begin{aligned}
\hat{\mathbf{v}}_{i}(t)=\gamma_{i}^{-1}[ & -\left(\nabla_{i} U\left(\mathbf{r}^{N}\right)\right)-k_{\mathrm{B}} T \nabla_{i} \\
& \left.-\left(\nabla_{i} V_{i}^{\mathrm{ext}}\left(\mathbf{r}_{i}, t\right)\right)+\mathbf{X}_{i}\left(\mathbf{r}_{i}, t\right)\right],
\end{aligned}
$$

where $\gamma_{i}$ is a friction constant related to the bare diffusion coefficient $D_{0 i}$ of species $i$, according to $\gamma_{i}=k_{\mathrm{B}} T / D_{0 i}$, with $k_{B}$ the Boltzmann constant and $T$ the temperature. In order to retain full generality, a particle dependent friction coefficient is considered. Note that only the thermal term is a differential operator; the two bracketed gradients each yield a vectorvalued function, which then acts via multiplication only. The derivative with respect to the position of particle $i$ in (2) acts on both the velocity operator and on the many-body distribution function. Physically, the action of $\hat{\mathbf{v}}_{i}(t)$ on the distribution, $\Psi\left(\mathbf{r}^{N}, t\right)$, generates the noise averaged velocity of particle $i$.

\subsection{One-body density and current}

The one-body density and one-body current of a tagged particle $i$ in a classical many-body system are respectively described by the following operators,

$$
\begin{gathered}
\hat{\rho}_{i}(\mathbf{r}, t)=\delta\left(\mathbf{r}-\mathbf{r}_{i}\right), \\
\hat{\mathbf{J}}_{i}(\mathbf{r}, t)=\delta\left(\mathbf{r}-\mathbf{r}_{i}\right) \hat{\mathbf{v}}_{i}(t),
\end{gathered}
$$


where $\hat{\mathbf{v}}_{i}(t)$ is the time-dependent velocity of particle $i$, given by (3). We consider the system to be a mixture consisting of $m$ species, labelled by $\alpha=1, \ldots, m$. Let $\mathcal{N}_{\alpha}$ be the set of particle indices $i$ that constitute species $\alpha$. Then the species-labelled density and current operators are given by

$$
\begin{aligned}
& \hat{\rho}_{\alpha}(\mathbf{r}, t)=\sum_{i \in \mathcal{N}_{\alpha}} \hat{\rho}_{i}(\mathbf{r}, t), \\
& \hat{\mathbf{J}}_{\alpha}(\mathbf{r}, t)=\sum_{i \in \mathcal{N}_{\alpha}} \hat{\mathbf{J}}_{i}(\mathbf{r}, t),
\end{aligned}
$$

The total density and current operators follow from summation over the species label

$$
\begin{aligned}
& \hat{\rho}(\mathbf{r}, t)=\sum_{\alpha} \hat{\rho}_{\alpha}(\mathbf{r}, t), \\
& \hat{\mathbf{J}}(\mathbf{r}, t)=\sum_{\alpha} \hat{\mathbf{J}}_{\alpha}(\mathbf{r}, t),
\end{aligned}
$$

where the sums run over all species, $\alpha=1, \ldots, m$.

The local conservation of tagged particle density is expressed by the one-body continuity equation

$$
\frac{\partial}{\partial t} \rho_{i}(\mathbf{r}, t)=-\nabla \cdot \mathbf{J}_{i}(\mathbf{r}, t)
$$

where the average tagged density and current are given by $\rho_{i}(\mathbf{r}, t)=\left\langle\hat{\rho}_{i}(\mathbf{r}, t)\right\rangle$ and $\mathbf{J}_{i}(\mathbf{r}, t)=\left\langle\hat{\mathbf{J}}_{i}(\mathbf{r}, t)\right\rangle$, respectively. Here the angles indicate an average with respect to the instantaneous distribution function, $\langle\cdot\rangle \equiv \int \mathrm{d} \mathbf{r}^{N} \cdot \Psi\left(\mathbf{r}^{N}, t\right)$. The continuity equation for the species resolved densities is obtained by summing (10) over all $i \in \mathcal{N}_{\alpha}$, and using the definitions (6) and (7), which yields

$$
\frac{\partial}{\partial t} \rho_{\alpha}(\mathbf{r}, t)=-\nabla \cdot \mathbf{J}_{\alpha}(\mathbf{r}, t),
$$

where the average density and current of species $\alpha$ are given by $\rho_{\alpha}(\mathbf{r}, t)=\left\langle\hat{\rho}_{\alpha}(\mathbf{r}, t)\right\rangle$ and $\mathbf{J}_{\alpha}(\mathbf{r}, t)=\left\langle\hat{\mathbf{J}}_{\alpha}(\mathbf{r}, t)\right\rangle$. Finally, summing over all particles in (10) yields the continuity equation for the total density

$$
\frac{\partial}{\partial t} \rho(\mathbf{r}, t)=-\nabla \cdot \mathbf{J}(\mathbf{r}, t)
$$

The average total velocity is given as the ratio $\mathbf{v}(\mathbf{r}, t)=$ $\mathbf{J}(\mathbf{r}, t) / \rho(\mathbf{r}, t)$. The average velocity of species $\alpha$ is $\mathbf{v}_{\alpha}(\mathbf{r}, t)=$ $\mathbf{J}_{\alpha}(\mathbf{r}, t) / \rho_{\alpha}(\mathbf{r}, t)$, and the average velocity of particle $i$ is $\mathbf{v}_{i}(\mathbf{r}, t)=\mathbf{J}_{i}(\mathbf{r}, t) / \rho_{i}(\mathbf{r}, t)$.

\section{Power functional theory for mixtures}

In equilibrium the central generating functional is the grand potential, which describes the free energy available to do work, subject to the contraints imposed on the system; namely fixed volume, temperature and chemical potential. For Brownian many-body systems out of equilibrium, it has been shown that the corresponding natural quantity is a 'free power' [14].
This dynamic generating functional is nonlocal in space and time and captures the interplay between reversible free energy changes and irreversible dissipative loss.

For a one-component system of Brownian particles it has been proven [14] that the free power of an arbitrary nonequilibrium state is a functional of the average onebody density and current. Separating off the external field contributions to the free power enables identification of an intrinsic contribution, which depends only on the interparticle interactions. The existence of an intrinsic free power, analogous to the intrinsic Helmholtz free energy in equilibrium density functional theory [18], is a powerful and highly nontrivial aspect of the PFT: For a given model interaction potential this enables approximations to be developed, which can then be employed without modification to systems subject to arbitrary external forces.

In order to implement the dynamical test particle method to be described below, we will require a generalization of the power functional method to treat mixtures. For multicomponent systems the free power, written as a sum of intrinsic and external field contributions, is given by the sum

$$
\begin{gathered}
\mathcal{R}_{t}\left[\left\{\rho_{\alpha}, \mathbf{J}_{\alpha}\right\}\right]=W_{t}\left[\left\{\rho_{\alpha}, \mathbf{J}_{\alpha}\right\}\right]+\sum_{\alpha} \int \mathrm{d} \mathbf{r} \dot{V}_{\alpha}^{\mathrm{ext}}(\mathbf{r}, t) \rho_{\alpha}(\mathbf{r}, t) \\
-\sum_{\alpha} \int \mathrm{d} \mathbf{r}\left(\mathbf{X}_{\alpha}(\mathbf{r}, t)-\nabla V_{\alpha}^{\mathrm{ext}}(\mathbf{r}, t)\right) \cdot \mathbf{J}_{\alpha}(\mathbf{r}, t),
\end{gathered}
$$

where $W_{t}$ is the intrinsic free power at time $t$, the overdot on the external potential denotes a partial derivative with respect to time, and the notation $\left[\left\{\rho_{\alpha}, \mathbf{J}_{\alpha}\right\}\right]$ indicates a functional dependence on the complete set of density and current fields, $\alpha=1, \ldots, m$.

The intrinsic free power may be subdivided into a sum of dissipative and adiabatic contributions

$$
W_{t}\left[\left\{\rho_{\alpha}, \mathbf{J}_{\alpha}\right\}\right]=P_{t}\left[\left\{\rho_{\alpha}, \mathbf{J}_{\alpha}\right\}\right]+\dot{F}\left[\left\{\rho_{\alpha}\right\}\right]
$$

where irreversible energy loss due to friction is described by the power dissipation functional, $P_{t}\left[\left\{\rho_{\alpha}, \mathbf{J}_{\alpha}\right\}\right]$, and adiabatic changes are captured by the (time derivative of the) equilibrium intrinsic Helmholtz free energy, $F\left[\left\{\rho_{\alpha}\right\}\right]$. The latter is a functional of the instantaneous density alone [18] and is taken to be known, either exactly or to a good level of approximation. We emphasise that the splitting (14) does not represent an approximation, but rather a physically motivated definition of $P_{t}\left[\left\{\rho_{\alpha}, \mathbf{J}_{\alpha}\right\}\right]$, expressing the expectation that free energy changes will be responsible for the adiabatic part of the dynamics.

In order to isolate the nontrivial many-body physics as cleanly as possible, we further split the dissipated power functional into two contributions

$P_{t}\left[\left\{\rho_{\alpha}, \mathbf{J}_{\alpha}\right\}\right]=P_{t}^{\mathrm{id}}\left[\left\{\rho_{\alpha}, \mathbf{J}_{\alpha}\right\}\right]+P_{t}^{\mathrm{exc}}\left[\left\{\rho_{\alpha}, \mathbf{J}_{\alpha}\right\}\right]$,

where the first term is the dissipation functional of a multicomponent ideal gas, given exactly by

$$
P_{t}^{\mathrm{id}}\left[\left\{\rho_{\alpha}, \mathbf{J}_{\alpha}\right\}\right]=\sum_{\alpha} \int \mathrm{d} \mathbf{r} \frac{\gamma_{\alpha} \mathbf{J}_{\alpha}(\mathbf{r}, t)^{2}}{2 \rho_{\alpha}(\mathbf{r}, t)},
$$


and the second term in (15) accounts for the nontrivial dissipation, including all memory effects, arising from interparticle interactions. Here the friction coefficient of species $\alpha$ is denoted by $\gamma_{\alpha}$.

For multicomponent systems the minimum principle (1) generalizes straightforwardly to the set of equations

$$
\frac{\delta \mathcal{R}_{t}\left[\left\{\rho_{\alpha^{\prime}}, \mathbf{J}_{\alpha^{\prime}}\right\}\right]}{\delta \mathbf{J}_{\alpha}(\mathbf{r}, t)}=0
$$

where $\alpha=1, \ldots, m$. Using equations (13)-(16) the EulerLagrange equation (17) can be written in the more explicit form

$$
\begin{aligned}
\frac{\gamma_{\alpha} \mathbf{J}_{\alpha}(\mathbf{r}, t)}{\rho_{\alpha}(\mathbf{r}, t)} & +\frac{\delta P_{t}^{\operatorname{exc}}\left[\left\{\rho_{\alpha^{\prime}}, \mathbf{J}_{\alpha^{\prime}}\right\}\right]}{\delta \mathbf{J}_{\alpha}(\mathbf{r}, t)} \\
& =-\nabla \frac{\delta F\left[\left\{\rho_{\alpha^{\prime}}\right\}\right]}{\delta \rho_{\alpha}(\mathbf{r}, t)}-\nabla V_{\alpha}^{\operatorname{ext}}(\mathbf{r}, t)+\mathbf{X}_{\alpha}(\mathbf{r}, t) .
\end{aligned}
$$

Given approximations for the free energy and the excess dissipation functionals, where the latter depends only on the interparticle interactions, substitution of (18) into the continuity equation (11) generates a closed set of coupled equations for the density fields.

The simplest (adiabatic) approximation within PFT is to neglect entirely the excess dissipation by setting $P_{t}^{\text {exc }}=0$. This recovers the well-known dynamical density functional theory (DDFT) [18-20]. Within this approximation, the density fields of different species are coupled solely via the Helmholtz free energy. In a more complete theory the equation (17) would be coupled (generally in a nonlinear fashion) by both the excess free energy and the excess dissipation functional.

\section{The van Hove function}

We return to one-component systems, and investigate these on the two-body level. The most commonly studied dynamical two-body correlation function is the densitydensity correlation function introduced by van Hove [1, 12]. For spatially and temporally inhomogeneous one-component systems this is given by

$$
G_{\mathrm{vH}}\left(\mathbf{r}_{1}, t_{1}, \mathbf{r}_{2}, t_{2}\right)=\frac{\left\langle\hat{\rho}\left(\mathbf{r}_{1}, t_{1}\right) \hat{\rho}\left(\mathbf{r}_{2}, t_{2}\right)\right\rangle}{\rho\left(\mathbf{r}_{1}, t_{1}\right)},
$$

where the average is taken using the many-body probability distribution at the earlier time, $t_{2}$, together with the manybody propagator that 'transports' the result of the action of the density operator at time $t_{2}$ to the later time $t_{1}$; [11] provides all details on the calculation of such two-time averages.

Generalizations of (19) exist for general multi-component systems [13]. However, in the present work we wish to focus on the dynamics of one-component systems. The species labelling introduced above will serve as a formal method to tag an arbitrarily chosen particle (which will serve to identify the self contribution). As a consequence the friction coefficient of tagged and distinct 'species' are identical, $\gamma_{\mathrm{s}}=\gamma_{\mathrm{d}} \equiv \gamma$. With this in mind, we split the total van Hove function (19) into a sum of two contributions

$G_{\mathrm{vH}}\left(\mathbf{r}_{1}, t_{1}, \mathbf{r}_{2}, t_{2}\right)=G_{\mathrm{vH}}^{\mathrm{s}}\left(\mathbf{r}_{1}, t_{1}, \mathbf{r}_{2}, t_{2}\right)+G_{\mathrm{vH}}^{\mathrm{d}}\left(\mathbf{r}_{1}, t_{1}, \mathbf{r}_{2}, t_{2}\right)$, where the self and the distinct part of the van Hove function is defined, correspondingly, by

$$
\begin{aligned}
& G_{\mathrm{vH}}^{\mathrm{s}}\left(\mathbf{r}_{1}, t_{1}, \mathbf{r}_{2}, t_{2}\right)=\sum_{i} \frac{\left\langle\hat{\rho}_{i}\left(\mathbf{r}_{1}, t_{1}\right) \hat{\rho}_{i}\left(\mathbf{r}_{2}, t_{2}\right)\right\rangle}{\rho\left(\mathbf{r}_{1}, t_{1}\right)}, \\
& G_{\mathrm{vH}}^{\mathrm{d}}\left(\mathbf{r}_{1}, t_{1}, \mathbf{r}_{2}, t_{2}\right)=\sum_{i k} \frac{\left\langle\hat{\rho}_{i}\left(\mathbf{r}_{1}, t_{1}\right) \hat{\rho}_{k}\left(\mathbf{r}_{2}, t_{2}\right)\right\rangle}{\rho\left(\mathbf{r}_{1}, t_{1}\right)} .
\end{aligned}
$$

The primed sum indicates a summation over distinct pairs, i.e. $\sum_{i k}^{\prime} \equiv \sum_{i} \sum_{k(\neq i)}$. The superscripts s and d, denoting the self and distinct contributions, can be formally regarded as labelling distinct species, despite the fact that all particles are physically identical.

For isotropic, translationally invariant bulk systems the van Hove functions (19), (21) and (22) become functions of the time difference $t \equiv t_{1}-t_{2}$ and of the radial distance $r=|\mathbf{r}|$, where $\mathbf{r} \equiv \mathbf{r}_{1}-\mathbf{r}_{2}$. The initial conditions at time $t=0$ are

$$
\begin{gathered}
G_{\mathrm{vH}}^{\mathrm{s}}(r, 0)=\delta(\mathbf{r}), \\
G_{\mathrm{vH}}^{\mathrm{d}}(r, 0)=\rho g(r),
\end{gathered}
$$

where $\delta(\mathbf{r})$ indicates a three-dimensional Dirac delta function (at the origin) and $g(r)$ is the equilibrium radial distribution function. The self and distinct intermediate scattering functions are given by the spatial Fourier transform

$$
\tilde{G}_{\mathrm{vH}}^{\alpha}(k, t)=\int \mathrm{d} \mathbf{r} G_{\mathrm{vH}}^{\alpha}(r, t) \mathrm{e}^{-\mathrm{i} \mathbf{k} \cdot \mathbf{r}},
$$

where $\alpha=\mathrm{s}$, d. Knowledge of the self intermediate scattering function provides direct access to the mean-squared particle displacement due to the diffusive motion via

$$
\left\langle r^{2}(t)\right\rangle=-\left.3 \frac{\mathrm{d}^{2}}{\mathrm{~d} k^{2}} \tilde{G}_{\mathrm{vH}}^{\mathrm{s}}(k, t)\right|_{k=0},
$$

where $\mathbf{r}=0$ at the start of the measurement, $t=0$, due to the use of the relative coordinates and the initial condition (23).

\section{Dynamic test particle limit}

The dynamic test particle limit, as first introduced by Archer et al [16], enables the self and distinct van Hove functions of a homogeneous one-component system in equilibrium to be determined from the density profiles of a binary mixture in an appropriately constructed inhomogeneous situation. The method is completely general and is applicable both in and outof-equilibrium. For the bulk in equilibrium at time $t=0$ the density profile of species $\mathrm{s}$ (self) is taken to be a delta function of unit norm located at the coordinate origin, $\rho_{\mathrm{s}}(\mathbf{r}, 0)=\delta(\mathbf{r})$, representing a tagged particle. The initial condition for species $\mathrm{d}$ (distinct) is given by $\rho_{\mathrm{d}}(\mathbf{r}, 0)=\rho g(r)$, where $\rho \equiv N / V$ is the bulk density and $g(r)$ is the bulk radial distribution function. If the particles are physically identical, such that the species labelling is just a formal device to keep track of the tagged particle, then the density profiles can be identified with the self 
and distinct van Hove functions of the one-component system, according to

$$
\begin{aligned}
& G_{\mathrm{vH}}^{\mathrm{s}}(r, t) \equiv \rho_{\mathrm{s}}(r, t), \\
& G_{\mathrm{vH}}^{\mathrm{d}}(r, t) \equiv \rho_{\mathrm{d}}(r, t) .
\end{aligned}
$$

In making the identifications (27) and (28) it is assumed that there are no external fields. However, the dynamic test particle approach remains valid in nonequilibrium. An example is e.g. shear flow, for which the system becomes anisotropic, but remains translationally invariant. In this case the functions appearing in (27) and (28) will simply acquire a vectorial argument $\mathbf{r}$ and are subject to the initial conditions $\rho_{\mathrm{s}}(\mathbf{r}, 0)=$ $\delta(\mathbf{r}), \rho_{\mathrm{d}}(\mathbf{r}, 0)=\rho g(\mathbf{r}, t)$, where $g(\mathbf{r})$ is the shear-distorted, steady state radial distribution function at the time $t$ (the shear field can be time-dependent).

The above arguments apply to translationally invariant systems. For completeness, we note that the most general situation is realized when the density becomes spatially inhomogeneous, $\rho(\mathbf{r}, t)$, as a result of either time-dependent external driving or external potential fields. In such cases equations (27) and (28) generalize to

$$
\begin{gathered}
G_{\mathrm{vH}}^{\mathrm{s}}\left(\mathbf{r}_{1}, t_{1}, \mathbf{r}_{2}, t_{2}\right) \equiv \rho_{\mathrm{s}}\left(\mathbf{r}_{1}, t_{1}\right), \\
G_{\mathrm{vH}}^{\mathrm{d}}\left(\mathbf{r}_{1}, t_{1}, \mathbf{r}_{2}, t_{2}\right) \equiv \rho_{\mathrm{d}}\left(\mathbf{r}_{1}, t_{1}\right),
\end{gathered}
$$

with the initial conditions $\rho_{\mathrm{s}}\left(\mathbf{r}_{1}, t_{2}\right)=\delta\left(\mathbf{r}_{1}-\mathbf{r}_{2}\right)$ and $\rho_{\mathrm{d}}\left(\mathbf{r}_{1}, t_{2}\right)=$ $\rho\left(\mathbf{r}_{1}, t_{2}\right) g\left(\mathbf{r}_{1}, \mathbf{r}_{2}, t_{2}\right)$, where the single-time inhomogeneous pair correlation function is related to the pair-density according to $g\left(\mathbf{r}_{1}, \mathbf{r}_{2}, t_{2}\right) \equiv \rho^{(2)}\left(\mathbf{r}_{1}, \mathbf{r}_{2}, t_{2}\right) /\left(\rho\left(\mathbf{r}_{1}, t_{2}\right) \rho\left(\mathbf{r}_{2}, t_{2}\right)\right)$.

\subsection{Self and distinct van Hove functions}

In the absence of external fields, combining (18) with the continuity equation (11) yields a coupled pair of equations for the self and distinct van Hove function

$\frac{\partial}{\partial t} G_{\mathrm{vH}}^{\alpha}(r, t)+\nabla \cdot\left[G_{\mathrm{vH}}^{\alpha}(r, t) \gamma^{-1} \mathbf{F}_{\alpha}^{\mathrm{tot}}(r, t)\right]=0$,

where $\alpha=\mathrm{s}, \mathrm{d}$ and the total force arising from interparticle interactions, thermal motion and interparticle dissipation is given by

$\mathbf{F}_{\alpha}^{\mathrm{tot}}(r, t)=-\nabla \frac{\delta F\left[\rho_{\mathrm{s}}, \rho_{\mathrm{d}}\right]}{\delta \rho_{\alpha}(\mathbf{r}, t)}-\frac{\delta P_{t}^{\mathrm{exc}}\left[\rho_{\mathrm{s}}, \rho_{\mathrm{d}}, \mathbf{J}_{\mathrm{s}}, \mathbf{J}_{\mathrm{d}}\right]}{\delta \mathbf{J}_{\alpha}(\mathbf{r}, t)}$.

The first term in (32) involves the total Helmholtz free energy, whereas the second term involves only the excess dissipation. The ideal dissipation term, which has here been been split off, generates the $\gamma \mathbf{J}_{\alpha} / \rho_{\alpha}$ term in (18) and thus leads, via the continuity equation, to the appearance of the time-derivative term in (31).

Interpreting $\gamma^{-1} \mathbf{F}_{\alpha}^{\mathrm{tot}}(r, t)$ as a generalized velocity field, the left hand side of equation (31) can be identified as a transport derivative acting on the van Hove functions. Equation (31) is highly nonlinear, as the density fields appearing in the functionals of (32) are related to the van Hove functions according to (27) and (28), while the current fields are obtained from the densities by solving equation (18). Although (31) appears to be symmetric with respect to species labels $\mathrm{s}$ and d, the asymmetry between the tagged particle and the others enters via the initial conditions and the explicit form of the generating functionals $F$ and $P_{t}^{\text {exc }}$.

The Helmholtz free energy can be split into a sum of ideal and excess contributions, $F\left[\left\{\rho_{\alpha}\right\}\right]=F^{\text {id }}\left[\left\{\rho_{\alpha}\right\}\right]+F^{\operatorname{exc}}\left[\left\{\rho_{\alpha}\right\}\right]$, where the former is given exactly by

$$
F^{\mathrm{id}}\left[\left\{\rho_{\alpha}\right\}\right]=k_{\mathrm{B}} T \sum_{\alpha} \int \mathrm{d} \mathbf{r} \rho_{\alpha}(\mathbf{r}, t)\left[\ln \left(\Lambda_{\alpha}^{3} \rho_{\alpha}(\mathbf{r}, t)\right)-1\right],
$$

where $\Lambda_{\alpha}$ is the (irrelevant) thermal de Broglie length of species $\alpha$, and in the present situation $\Lambda_{\mathrm{s}}=\Lambda_{\mathrm{d}} \equiv \Lambda$. The excess contribution depends upon the interparticle interactions of the specific model under consideration. Employing this splitting in (31) enables the interaction contributions to be separated off,

$$
\begin{aligned}
\frac{\partial}{\partial t} G_{\mathrm{vH}}^{\alpha}(r, t)= & D_{0} \nabla^{2} G_{\mathrm{vH}}^{\alpha}(r, t) \\
& -\nabla \cdot\left[G_{\mathrm{vH}}^{\alpha}(r, t) \gamma^{-1} \mathbf{F}_{\alpha}^{\mathrm{int}}(r, t)\right]=0,
\end{aligned}
$$

where the forces arising from interparticle interactions are given by

$$
\mathbf{F}_{\alpha}^{\mathrm{int}}(r, t)=-\nabla \frac{\delta F^{\mathrm{exc}}\left[\left\{\rho_{\alpha^{\prime}}\right\}\right]}{\delta \rho_{\alpha}(\mathbf{r}, t)}-\frac{\delta P_{t}^{\operatorname{exc}}\left[\left\{\rho_{\alpha^{\prime}}, \mathbf{J}_{\alpha^{\prime}}\right\}\right]}{\delta \mathbf{J}_{\alpha}(\mathbf{r}, t)} .
$$

In contrast to the adiabatic free energy term, the dissipative contributions to the interaction force are nonconservative.

\subsection{Intermediate scattering functions}

Experiments can provide results for the self and distinct intermediate scattering functions, which are here obtained by spatial Fourier transformation of (34) to yield

$$
\begin{aligned}
\frac{\partial}{\partial t} \quad \tilde{G}_{\mathrm{vH}}^{\alpha}(k, t) & =-D_{0} k^{2} \tilde{G}_{\mathrm{vH}}^{\alpha}(k, t) \\
& +\frac{\mathrm{ik}}{8 \pi^{3} \gamma} \cdot \int \mathrm{d} \mathbf{k}^{\prime} \tilde{G}_{\mathrm{vH}}^{\alpha}\left(\left|\mathbf{k}-\mathbf{k}^{\prime}\right|, t\right) \tilde{\mathbf{F}}_{\alpha}^{\mathrm{int}}\left(k^{\prime}, t\right) .
\end{aligned}
$$

where the Fourier transformed force, $\tilde{\mathbf{F}}_{\alpha}^{\text {int }}(k, t)$, is purely imaginary. The relation (36) constitutes an exact pair of equations for the self and distinct intermediate scattering function.

The species labelled one-body direct correlation functions are defined in terms of the excess free energy according to [18]

$$
c_{\alpha}^{(1)}(\mathbf{r}) \equiv-\left(k_{\mathrm{B}} T\right)^{-1} \frac{\delta F^{\mathrm{exc}}\left[\left\{\rho_{\alpha^{\prime}}\right\}\right]}{\delta \rho_{\alpha}(\mathbf{r})},
$$

which, for a bulk system, is related to the excess chemical potential by $c_{\alpha, \text { bulk }}^{(1)}=-\mu_{\alpha}^{\text {exc }} / k_{\mathrm{B}} T$. Substitution of (35) and (37) into equation (36) enables the equation of motion to be recast in the form

$$
\begin{aligned}
& \frac{\partial}{\partial t} \tilde{G}_{\mathrm{vH}}^{\alpha}(k, t)=-D_{0} k^{2} \tilde{G}_{\mathrm{vH}}^{\alpha}(k, t) \\
& +\frac{D_{0}}{8 \pi^{3}} \int \mathrm{d} \mathbf{k}^{\prime} \mathbf{k} \cdot \mathbf{k}^{\prime} \tilde{G}_{\mathrm{vH}}^{\alpha}\left(\left|\mathbf{k}-\mathbf{k}^{\prime}\right|, t\right) \tilde{c}_{\alpha}^{(1)}\left(k^{\prime}, t\right) \\
& \quad+\frac{\mathrm{i}}{8 \pi^{3} \gamma} \int \mathrm{d} \mathbf{k}^{\prime} \tilde{G}_{\mathrm{vH}}^{\alpha}\left(\left|\mathbf{k}-\mathbf{k}^{\prime}\right|, t\right) \mathbf{k} \cdot \tilde{\mathbf{F}}_{\alpha}^{\mathrm{diss}}\left(k^{\prime}, t\right),
\end{aligned}
$$


where the last term involves the Fourier transform of the superadiabatic force (also known as the irreducible force)

$$
\mathbf{F}_{\alpha}^{\mathrm{diss}}(r, t)=-\frac{\delta P_{t}^{\operatorname{exc}}\left[\left\{\rho_{\alpha^{\prime}}, \mathbf{J}_{\alpha^{\prime}}\right\}\right]}{\delta \mathbf{J}_{\alpha}(\mathbf{r}, t)}
$$

which accounts for the dissipation arising from interparticle interactions.

\subsection{RY approximation to the free energy}

Ramakrishnan and Youssouff (RY) suggested an approximation to the excess free energy, valid for small density inhomogeneities, by considering a functional Taylor expansion about the homogeneous state and truncating at quadratic order. For a mixture this is given by [21]

$$
\begin{gathered}
F^{\mathrm{exc}}\left[\left\{\rho_{\alpha}\right\}\right]=F^{\mathrm{exc}}\left[\left\{\rho_{\alpha}^{\mathrm{bulk}}\right\}\right]+\sum_{\alpha} \mu_{\alpha}^{\mathrm{exc}} \int \mathrm{d} \mathbf{r} \bar{\rho}_{\alpha}(\mathbf{r}) \\
-\frac{k_{\mathrm{B}} T}{2} \sum_{\alpha \beta} \int \mathrm{d} \mathbf{r} \int \mathrm{d} \mathbf{r}^{\prime} c_{\alpha \beta}^{(2)}\left(\left|\mathbf{r}-\mathbf{r}^{\prime}\right|\right) \bar{\rho}_{\alpha}(\mathbf{r}) \bar{\rho}_{\beta}\left(\mathbf{r}^{\prime}\right),
\end{gathered}
$$

where $\bar{\rho}_{\alpha}(\mathbf{r})=\rho_{\alpha}(\mathbf{r})-\rho_{\alpha}^{\text {bulk }}$ is the deviation of the inhomogeneous density profile from the constant bulk density. For general inhomogeneous equilibrium situations the pair direct correlation between species $\alpha$ and $\beta$ is defined by the functional derivative

$$
c_{\alpha \beta}^{(2)}\left(\mathbf{r}, \mathbf{r}^{\prime}\right) \equiv \frac{\delta c_{\alpha}^{(1)}(\mathbf{r})}{\delta \rho_{\beta}\left(\mathbf{r}^{\prime}\right)},
$$

This two-point correlation function is the convolution kernel in (40) that acts as an effective potential coupling the density fluctuations. Note that the pair direct correlation depends only on a single scalar argument, as the functional Taylor expansion is performed around the homogeneous bulk fluid.

Within the approximation (40) the one- and two-body direct correlation functions of a mixture are related by a convolution integral

$$
c_{\alpha}^{(1)}(\mathbf{r})=\sum_{\beta} \int \mathrm{d} \mathbf{r}^{\prime} c_{\alpha \beta}^{(2)}\left(\left|\mathbf{r}-\mathbf{r}^{\prime}\right|\right) \bar{\rho}_{\beta}\left(\mathbf{r}^{\prime}\right) .
$$

However, for the dynamic test particle limit under consideration in this work the 'mixture' consists of a bath of particles of species d and a single tagged particle, species s. Both species are physically identical. The pair direct correlation functions are thus independent of the species labels, $c_{\alpha \beta}^{(2)}\left(\left|\mathbf{r}-\mathbf{r}^{\prime}\right|\right) \equiv c^{(2)}\left(\left|\mathbf{r}-\mathbf{r}^{\prime}\right|\right)$, as can be deduced from the definition (41). Furthermore, it is possible at this stage to incorporate the physical requirement that the tagged particle does not interact with itself [16], by imposing that $c_{\mathrm{ss}}^{(2)}(\mid \mathbf{r}-$ $\left.\mathbf{r}^{\prime} \mid\right)=0$. This physical input breaks the symmetry of the free energy (40) with respect to the self and distinct species.

\subsection{RY intermediate scattering functions}

Using (27) and (28) in (42) and assuming the spherical symmetry appropriate for the present dynamic test particle situation in a bulk fluid yields approximate one-body direct correlation functions

$$
\begin{aligned}
& c_{\mathrm{d}}^{(1)}(r, t)=\int \mathrm{d} \mathbf{r}^{\prime} c^{(2)}\left(\left|\mathbf{r}-\mathbf{r}^{\prime}\right|\right) H_{\mathrm{vH}}\left(r^{\prime}, t\right), \\
& c_{\mathrm{s}}^{(1)}(r, t)=\int \mathrm{d} \mathbf{r}^{\prime} c^{(2)}\left(\left|\mathbf{r}-\mathbf{r}^{\prime}\right|\right) H_{\mathrm{vH}}^{\mathrm{d}}\left(r^{\prime}, t\right),
\end{aligned}
$$

where we have introduced the shifted functions

$$
\begin{aligned}
& H_{\mathrm{vH}}^{\mathrm{d}}(r, t)=G_{\mathrm{vH}}^{\mathrm{d}}(r, t)-\rho, \\
& H_{\mathrm{vH}}(r, t)=G_{\mathrm{vH}}(r, t)-\rho,
\end{aligned}
$$

which have the property that they tend to zero at large separations $\left(G_{\mathrm{vH}}^{\mathrm{s}}(r, t)\right.$ already does this). In equations (43) and (44) we have eliminated the unphysical self-self interaction and employed the fact that $\rho_{\mathrm{s}}=0$ and $\rho_{\mathrm{d}}=\rho \equiv N / V$ in the thermodynamic limit.

Substitution of (43) and (44) into (38) generates (approximate) coupled equations for the distinct van Hove function,

$$
\begin{aligned}
& \frac{\partial}{\partial t} \tilde{G}_{\mathrm{vH}}^{\mathrm{d}}(k, t)=-D_{0} k^{2} \tilde{G}_{\mathrm{vH}}^{\mathrm{d}}(k, t) \\
& +\frac{D_{0}}{8 \pi^{3}} \int \mathrm{d} \mathbf{k}^{\prime} \mathbf{k} \cdot \mathbf{k}^{\prime} \tilde{G}_{\mathrm{vH}}^{\mathrm{d}}\left(\left|\mathbf{k}-\mathbf{k}^{\prime}\right|, t\right) \tilde{c}^{(2)}\left(k^{\prime}, t\right) \tilde{G}_{\mathrm{vH}}\left(k^{\prime}, t\right) \\
& \quad+\frac{\mathrm{i}}{8 \pi^{3} \gamma} \int \mathrm{d} \mathbf{k}^{\prime} \tilde{G}_{\mathrm{vH}}^{\mathrm{d}}\left(\left|\mathbf{k}-\mathbf{k}^{\prime}\right|, t\right) \mathbf{k} \cdot \tilde{\mathbf{F}}_{\mathrm{d}}^{\mathrm{diss}}\left(k^{\prime}, t\right),
\end{aligned}
$$

and for the self van Hove function,

$$
\begin{aligned}
& \frac{\partial}{\partial t} \tilde{G}_{\mathrm{vH}}^{\mathrm{s}}(k, t)=-D_{0} k^{2} \tilde{G}_{\mathrm{vH}}^{\mathrm{s}}(k, t) \\
& +\frac{D_{0}}{8 \pi^{3}} \int \mathrm{d} \mathbf{k}^{\prime} \mathbf{k} \cdot \mathbf{k}^{\prime} \tilde{G}_{\mathrm{vH}}^{\mathrm{s}}\left(\left|\mathbf{k}-\mathbf{k}^{\prime}\right|, t\right) \tilde{c}^{(2)}\left(k^{\prime}, t\right) \tilde{G}_{\mathrm{vH}}^{\mathrm{d}}\left(k^{\prime}, t\right) \\
& +\frac{\mathrm{i}}{8 \gamma \pi^{3}} \int \mathrm{d} \mathbf{k}^{\prime} \tilde{G}_{\mathrm{vH}}^{\mathrm{s}}\left(\left|\mathbf{k}-\mathbf{k}^{\prime}\right|, t\right) \mathbf{k} \cdot \tilde{\mathbf{F}}_{\mathrm{s}}^{\mathrm{diss}}\left(k^{\prime}, t\right) .
\end{aligned}
$$

The distinct equation (47) is, with the exception of the dissipative force term, structurally identical to an expression previously employed in a DDFT study of the intermediate stages of spinodal decomposition (see equation (37) of [19]). However, the physical interpretation here is very different to that of [19], where DDFT was used to calculate the time evolution of the one-component density following a quench into the two-phase region. Via (27) and (28) we relate the density fields of a mixture to the two-body correlation functions.

The distinct equation (47) can be expressed as

$$
\begin{aligned}
& \frac{\partial}{\partial t} \tilde{G}_{\mathrm{vH}}^{\mathrm{d}}(k, t)=-\left(\frac{D_{0} k^{2}}{\mathrm{~S}(k, t)}\right) \tilde{G}_{\mathrm{vH}}^{\mathrm{d}}(k, t) \\
& +\frac{D_{0}}{8 \pi^{3}} \int \mathrm{d} \mathbf{k}^{\prime} \mathbf{k} \cdot \mathbf{k}^{\prime} \tilde{H}_{\mathrm{vH}}^{\mathrm{d}}\left(\left|\mathbf{k}-\mathbf{k}^{\prime}\right|, t\right) \tilde{c}^{(2)}\left(k^{\prime}, t\right) \tilde{H}_{\mathrm{vH}}\left(k^{\prime}, t\right), \\
& +\frac{\mathrm{i}}{8 \pi^{3} \gamma} \int \mathrm{d} \mathbf{k}^{\prime} \tilde{G}_{\mathrm{vH}}^{\mathrm{d}}\left(\left|\mathbf{k}-\mathbf{k}^{\prime}\right|, t\right) \mathbf{k} \cdot \tilde{\mathbf{F}}_{\mathrm{d}}^{\mathrm{diss}}\left(k^{\prime}, t\right),
\end{aligned}
$$

where the time-dependent function $\mathrm{S}(k, t)$ is given by

$\mathrm{S}(k, t) \equiv S(k)\left(1-(S(k)-1)\left(\frac{\tilde{G}_{\mathrm{vH}}^{\mathrm{s}}(k, t)}{\tilde{G}_{\mathrm{vH}}^{\mathrm{d}}(k, t)}\right)\right)^{-1}$. 
The static structure factor is related to the direct correlation function by $S(k)=\left(1-\rho^{\text {bulk }} c^{(2)}(k)\right)^{-1}$. It is not neccessary to rewrite equation (48) for the self van Hove function, because one can simply replace $\tilde{G}_{\mathrm{vH}}^{\mathrm{d}}\left(k^{\prime}, t\right)$ by $\tilde{H}_{\mathrm{vH}}^{\mathrm{d}}\left(k^{\prime}, t\right)$ in the given expression (an additional delta function generated by the constant bulk density in (45) gives zero contibution to the integral in (48)).

\subsection{Mean-squared displacement}

The mean squared displacement of the tagged particle can now be obtained from the low wavevector limit of the self van Hove equation (48). Substitution of (48) into (26) generates an expression for the mean squared displacement

$$
\begin{aligned}
& \frac{\partial}{\partial t}\left\langle r^{2}(t)\right\rangle=6 D_{0}-\frac{3 D_{0}}{4 \pi^{3}} \int \mathrm{d} \mathbf{k}^{\prime} \frac{\partial \tilde{G}_{\mathrm{vH}}^{\mathrm{s}}\left(k^{\prime}, t\right)}{\partial k^{\prime}} \\
& \times \hat{\mathbf{k}} \cdot\left(\mathbf{k}^{\prime} \tilde{c}\left(k^{\prime}\right) \tilde{G}_{\mathrm{vH}}^{\mathrm{d}}\left(k^{\prime}, t\right)+\frac{\mathrm{i}}{k_{\mathrm{B}} T} \tilde{\mathbf{F}}_{\mathrm{s}}^{\mathrm{diss}}\left(k^{\prime}, t\right)\right),
\end{aligned}
$$

where $\hat{\mathbf{k}}$ is a unit vector. The first term generates simple free diffusion of the tagged particle, whereas the integral term describes the interaction of the tagged particle with its environment, consisting of adiabatic and superadiabatic contributions.

\section{6. de Gennes' approximation}

Truncation of both equations (48) and (49) at the first term provides the simplest approximation to the van Hove functions

$$
\begin{gathered}
\frac{\partial}{\partial t} \tilde{G}_{\mathrm{vH}}^{\mathrm{s}}(k, t)=-D_{0} k^{2} \tilde{G}_{\mathrm{vH}}^{\mathrm{s}}(k, t), \\
\frac{\partial}{\partial t} \tilde{G}_{\mathrm{vH}}^{\mathrm{d}}(k, t)=-\frac{D_{0} k^{2}}{\mathrm{~S}(k, t)} \tilde{G}_{\mathrm{vH}}^{\mathrm{d}}(k, t),
\end{gathered}
$$

the first of which trivially predicts free diffusion of the self van Hove function

$$
\tilde{G}_{\mathrm{vH}}^{\mathrm{s}}(k, t)=\exp \left(-D_{0} k^{2} t\right)
$$

Substitution of equations (52) and (53) into the exact splitting

$$
\tilde{G}_{\mathrm{vH}}(k, t)=\tilde{G}_{\mathrm{vH}}^{\mathrm{s}}(k, t)+\tilde{G}_{\mathrm{vH}}^{\mathrm{d}}(k, t),
$$

yields an approximation to the full van Hove function

$$
\tilde{G}_{\mathrm{vH}}(k, t)=S(k) \exp \left(-\frac{D_{0} k^{2}}{S(k)} t\right) .
$$

Equation (56) is a well known approximation, first proposed by de Gennes in 1959 [22]. This describes the relaxation of the van Hove function as a free diffusion process, but with an effective, wavevector dependent diffusion constant $D_{0} / S(k)$. Relaxation is thus slowest for wavevectors around the main peak of the structure factor, corresponding to the length-scale set by the nearest neighbour particles.

The truncation of equations (48) and (49) at the first term thus provides a consistent description of the self and distinct particle motion on the level of the de Gennes approximation
(56). The next step beyond this is to include the second (integral) terms on the right hand side of (48) and (49), which require no additional input information to evaluate. This adiabatic approximation effectively sets $P_{t}^{\operatorname{exc}}[\rho, \mathbf{J}]=0$, such that the excess dissipation force (39) vanishes. Going beyond the adiabatic approximation, then requires an expression for $P_{t}^{\operatorname{exc}}[\rho, \mathbf{J}]$ to provide a closed theory.

\section{Concluding remarks}

We have demonstrated how the power functional framework [14] can be applied to develop an exact dynamical test particle theory for the self and distinct parts of the van Hove function of a bulk one-component system. The first step in this development is the generalization of the power functional to treat mixtures of different particle species. The availability of a mixture theory is an essential requirement for implementation of the dynamic test particle limit, as this requires that a tagged particle can be identified by its species label. Application of the dynamic test particle limit then generated exact equations of motion (38) for the van Hove functions, which we expressed in Fourier space and related to existing approximation schemes. The present work builds upon that of Archer et al $[16,19]$ by formulating the dynamic test particle method within the framework of PFT.

The exact equations of motion (38) for the van Hove functions require as input the excess Helmholtz free energy functional, assumed to be known, and the excess power dissipation functional, which remains unspecified. Even at the lowest (adiabatic) order of approximation, for which the excess dissipation is neglected, the theory goes beyond the de Gennes' level of approximation by including a coupling between different wavevectors (modes). Archer et al [16] found that equations of this type provide qualitatively reasonable results for the van Hove functions at low and intermediate densities. Improving the description further will require carefully constructed approximations for the excess dissipation functional. Some physically motivated approximation schemes were suggested in [14], however, the search for accurate approximations to the excess dissipation remains a highly nontrivial task and work in this direction is ongoing.

The present development is related to the mode-coupling theory of liquid dynamics [8], which provides approximate equations of motion for the self and distinct intermediate scattering functions. In common with the mode-coupling approach, equations (48) and (49) couple the dynamics at different wavevectors and involve a history dependence, which enters here via the excess dissipation functional. As mentioned in the introduction, an alternative approach to the (total) intermediate scattering function is provided by the nonequilibrium Ornstein-Zernike (NOZ) equation [10,11]. In this case the structural similarities between the NOZ equation and the mode-coupling equation of motion are explicit. It would be interesting to explore these connections further within the present test-particle formulation. Moreover, given that mode-coupling theory can also be derived from field theoretic methods [23], it may prove fruitful to investigate 
the relation of the present approach to nonequilibrium field theories.

We emphasize that both the dynamic test particle limit and the PFT are very general methods, which can be applied in and out-of-equilibrium. These approaches are well-suited to treat spatial inhomogeneity arising from external fields or transient relaxation and remain valid in the presence of external driving (e.g. externally applied shear flow).

\section{References}

[1] Hansen J P and McDonald I R 2006 Theory of Simple Liquids 3rd edn (London: Academic)

[2] Green M S 1954 J. Chem. Phys. 22398

Kubo R 1957 J. Phys. Soc. Japan 12570

[3] Kubo R, Toda M and Hashitsumi N 1991 Stastical Physics II: Nonequilibrium Statistical Mechanics (Berlin: Springer)

[4] Zwanzig R 1961 Lectures in Theoretical Physics vol 3, ed W E Britton et al (New York: Wiley)

[5] Zwanzig R 2001 Nonequilibrium Statistical Mechanics (Oxford: Oxford University Press)

[6] Grabert H 1982 Projection Operator Techniques in Nonequilibrium Statistical Mechanics (Berlin: Springer)
[7] Chung C-H and Yip S 1969 Phys. Rev. 182323

[8] Götze W 2009 Complex Dynamics of Glass-Forming Liquids: a Mode-Coupling Theory (Oxford: Oxford University Press)

[9] Ramshaw J D 1981 Phys. Rev. A 241567

[10] Brader J M and Schmidt M 2013 J. Chem. Phys. 139104108

[11] Brader J M and Schmidt M 2014 J. Chem. Phys. 140034104

[12] van Hove L 1954 Phys. Rev. 95249

[13] Chávez-Rojo M A and Medina-Noyola M 2006 Physica A 36655

[14] Schmidt M and Brader J M 2013 J. Chem. Phys. 138214101

[15] Percus J K 1962 Phys. Rev. Lett. 8462

[16] Archer A J, Hopkins P and Schmidt M 2007 Phys. Rev. E 75040501

Hopkins P, Fortini A, Archer A J and Schmidt M $2010 \mathrm{~J}$. Chem. Phys. 133224505

[17] Dhont J K G 1996 An Introduction to the Dynamics of Colloids (Amsterdam: Elsevier)

[18] Evans R 1979 Adv. Phys. 28143

[19] Archer A J and Evans R 2004 J. Chem. Phys. 1214246

[20] Marini U and Tarazona P 1999 J. Chem. Phys. 1108032

[21] Ramakrishnan T V and Youssouff M 1979 Phys. Rev. B 192775

[22] de Gennes P-G 1959 Physica 25825

[23] Jacquin H and van Wijland F 2011 Phys. Rev. Lett. 106210602 